\title{
Palomar 13: A velocity dispersion inflated by binaries?`
}

\author{
A. Blecha ${ }^{1}$, G. Meylan ${ }^{2, \star \star}$, P. North ${ }^{3}$, and F. Royer ${ }^{1}$ \\ 1 Observatoire de Genève, Université de Genève, Chemin des Maillettes 51, 1290 Sauverny, Switzerland \\ e-mail: frederic.royer@obs.unige.ch \\ 2 Space Telescope Science Institute, 3700 San Martin Drive, Baltimore, MD 21218, USA \\ e-mail: gmeylan@stsci.edu \\ ${ }^{3}$ Laboratoire d'Astrophysique, École Polytechnique Fédérale de Lausanne, Observatoire, 1290 Chavannes-des-Bois, \\ Switzerland \\ e-mail: Pierre.North@obs.epfl.ch
}

Received 14 January 2004 / Accepted 19 February 2004

\begin{abstract}
Recently, combining radial velocities from Keck/HIRES échelle spectra with published proper motion membership probabilities, Côté et al. (2002) observed a sample of 21 stars, probable members of Palomar 13, a globular cluster in the Galactic halo. Their projected velocity dispersion $\sigma_{\mathrm{p}}=2.2 \pm 0.4 \mathrm{~km} \mathrm{~s}^{-1}$ gives a mass-to-light ratio $\mathcal{M} / L_{V}=40_{-17}^{+24}$, about one order of magnitude larger than the usual estimate for globular clusters. We present here radial velocities measured from three different CCD frames of commissioning observations obtained with the new ESO/VLT instrument FLAMES (Fibre Large Array Multi Element Spectrograph). From these data, now publicly available, we measure the homogeneous radial velocities of eight probable members of this globular cluster. A new projected velocity dispersion $\sigma_{\mathrm{p}}=0.6-0.9 \pm 0.3 \mathrm{~km} \mathrm{~s}^{-1}$ implies Palomar 13 mass-to-light ratio $\mathcal{M} / L_{V}=3-7$, similar to the usual value for globular clusters. We discuss briefly the two most obvious reasons for the previous unusual mass-to-light ratio finding: binaries, now clearly detected, and more homogeneous data from the multi-fibre FLAMES spectrograph.
\end{abstract}

Key words. Galaxy: globular clusters: individual: Palomar 13 - techniques: radial velocities - stars: kinematics stars: population II

\section{Introduction}

All the dynamical studies of nearby globular clusters have established that these dynamical systems contain no dark matter, apart from the expected stellar remnants such as white dwarves and neutron stars (e.g., Pryor \& Meylan 1993; Meylan \& Heggie 1997). Consequently, globular clusters may be the most massive stellar systems in which no non-baryonic dark matter is dynamically detected, while dynamical evidence for non-baryonic dark matter seems to be present in most galaxies, from the faintest dwarf spheroidals (dSphs) to the brightest $\mathrm{cD}$ galaxies, and clusters of galaxies as well.

Some of the local dSphs, around the Galaxy and M 31, have integrated absolute luminosities similar or fainter than those of the brightest Galactic globular clusters. Since there is evidence that some Galactic dSph galaxies are dark-matter-dominated,

\footnotetext{
Send offprint requests to: A. Blecha, e-mail: andre.blecha@obs.unige.ch

* Tables 2 and 3 are also available in electronic form at the CDS via anonymous ftp to cdsarc.u-strasbg.fr $(130.79 .128 .5)$ or via http://cdsweb.u-strasbg.fr/cgi-bin/qcat?]/A+A/419/533 $\star \star$ Affiliated with the Space Telescope Division of the European Space Agency, ESTEC, Noordwijk, The Netherlands.
}

it is therefore reasonable to check if some globular clusters do present dynamical evidence for non-baryonic dark matter. Such a possibility may be emphasized by the current predictions of Cold Dark Matter (CDM) numerical simulations of galaxy formation, in which the number of low-mass dark-matter substructures orbiting the halo of massive galaxies largely exceeds the number of dwarf galaxies observed in the halos of both our Galaxy and M31 (e.g., Klypin et al. 1999; Moore et al. 1999, 2001). However, recently improved CDM models may correctly predict the observed number of satellite galaxies (Binney 2003).

Could some of the globular clusters in the outer parts of the Galactic halo be such dark-matter substructure? These remote stellar systems have so far been poorly studied because of the difficulties in the acquisition of high-quality radial velocities and proper motions, direct consequences of the faintness and sparsity of these distant stellar systems. They are nevertheless important probes of the formation and evolution of the Galaxy, as their ages and metallicities provide direct constraints on the duration of halo formation process and on the time-scale for Galactic chemical enrichment, while the shape and extent of the Galactic dark halo are constrained by their orbital properties. 
In 1998, a program was started at Californian Institute of Technology to study the internal dynamics of seven distant halo globular clusters using the High Resolution Echelle Spectrometer (HIRES) at the W. M. Keck Observatory. The aim was the first direct measurements of the velocity dispersions and mass-to-light ratios for these clusters. Six clusters in this sample exhibited velocity dispersions $\sigma_{\mathrm{p}} \sim 1 \mathrm{~km} \mathrm{~s}^{-1}$, translating into mass-to-light ratio values typical of globular clusters $\mathcal{M} / L_{V} \sim 3$ (all mass-to-light ratios quoted in this paper are in solar units). Only one cluster, the halo globular cluster Palomar 13, displayed a velocity dispersion larger than expected.

Côté et al. (2002, also referenced below as the Keck study) presented a careful analysis, combining radial velocities from Keck/HIRES échelle spectra with published proper motion membership probabilities from Siegel et al. (2001). They obtained a sample of 21 stars, probable members of Palomar 13. Their projected, intrinsic velocity dispersion of $\sigma_{\mathrm{p}}=2.2 \pm$ $0.4 \mathrm{~km} \mathrm{~s}^{-1}$ implied a mass-to-light ratio $\mathcal{M} / L_{V}=40_{-17}^{+24}$, about one order of magnitude larger than the usual value for globular clusters. Côté et al. discussed at length all possible reasons for such an unusual result: (i) some velocity "jitter" among the red giants; (ii) a few binary stars; (iii) a non-standard mass function; (iv) process of dissolving into the Galactic halo through catastrophic tidal heating during a recent perigalacticon passage; or (v) the presence of a massive non-baryonic dark matter halo.

It is worth emphasizing that, in Côté et al., careful determination of the error bars made the usual mass-to-light ratio values for globular clusters $\mathcal{M} / L_{V} \sim 3$ at about two sigmas from the $\mathcal{M} / L_{V}$ value obtained for Palomar 13 .

Because of this marginally significant and puzzling result from Côté et al., some more spectroscopic data of stars in the field of Palomar 13 were acquired during the commissioning of the ESO/VLT instrument FLAMES. We present hereafter new high-quality and homogeneous radial velocities for 46 stars, 9 of them being members of Palomar 13, which provide new velocity dispersion and mass-to-light ratio values for this globular cluster. The remaining of this paper is as follows: Sect. 2 presents the observation and the data reduction, Sect. 3 discusses the membership of the stars, Sect. 4 gives the new velocity dispersion and corresponding mass-to-light ratio, and Sect. 5 discusses the plausible reasons for the difference between the present results and those obtained by Côté et al.

\section{Observations and data reduction}

\subsection{Observations}

All the new data presented in this paper were acquired between August 28 and September 3 2002, during the commissioning of the ESO/VLT instrument FLAMES (Fibre Large Array Multi Element Spectrograph), at Paranal, Chile. For more information on this instrument, see the ESO web page http://www . eso. org and the recent publications by Pasquini et al. (2002) and Royer et al. (2002). In order to clarify some possibly confusing uses of denominations, it is worth mentioning that FLAMES is the name of the instrument, while GIRAFFE is the name of the spectrograph within FLAMES.

We were involved neither in the selection of the stars nor in the preparation and acquisition of these observations. Recently, these data were made publicly available from the ESO/VLT archives site ${ }^{1}$.

The Palomar 13 observations are summarized in Table 1. Three exposures, each of one hour integration, were acquired in early September 2002. Calibration files were acquired a few days earlier. All data were taken in the high-resolution spectrograph setup HR-9 with spectral domain spanning from 514.3 to $535.4 \mathrm{~nm}$ and resolution $\lambda / \delta \lambda \sim 20000$. Both positioning plates of MEDUSA were used with the 133 fibres assigned as follows: 55 fibres to program stars, 14 to sky measurements, 5 to simultaneous wavelength calibrations, while 59 fibres remained unused. Average signal-to-noise ratio (SNR) for stars with $V=18$ is given as indication of spectra exposure. Note that expected accuracy for HR-9 setup and $S N R=10$ is $\sigma_{V_{\mathrm{r}}}=0.20 \mathrm{~km} \mathrm{~s}^{-1}$ (see Royer et al. 2002).

\subsection{Data reduction and calibration}

For each positioning plate, two calibration frames were used. The first one - the flat field - gave the master localization and analytical model of the PSF perpendicular to the spectral dispersion direction, while the second one - ThAr wavelength calibration frame - provided two pieces necessary for the master wavelength solution: the 2-D optical solution and the 2-D Chebyshev polynomial correction. For the HR-9 setup, approximately 6000 line positions were used to adjust the 30 parameters of the solution. Finally, the rebinned extracted ThAr spectra were cross-correlated with the ThAr mask and the measured mismatch between spectra was used to update the slit geometry model.

The spectra were extracted using the standard Python version of BLDRS - Baseline Data Reduction Software (girbldrs-1.09 and girbldrs-pipe-1.05) available from http://girbldrs. sourceforge. net. Basic description of BLDRS is given in Blecha et al. (2000).

The most important calibration feature of GIRAFFE is the presence of five Simultaneous Wavelength Calibration (SIMCAL) ThAr spectra in each exposure. The SIMCAL spectra are optimally exposed and regularly spaced over the detector (spectra $\# 1,32,63,94$, and 125 out of 133 in the present setup). They are used to accurately adjust the master localization and the master wavelength calibration.

The raw images were processed through the following steps: bias subtraction, localization adjustment, optimal extraction, rebinning to linear wavelength space with step of $0.005 \mathrm{~nm}$ using the master wavelength solution, wavelength solution translation using the SIMCAL spectra cross-correlation with ThAr mask and the final rebinning using the translated wavelength solution. Note that we did not subtract dark (negligible effects) and we did not flat-field (irrelevant for the cross-correlation), the flat-field frame being only used to derive

\footnotetext{
${ }^{1}$ http://www.eso.org/science/flames_comm/ FLAMES_comm_PAL_13.html
} 
Table 1. Log-book of the observations from FLAMES commissioning data archives: Palomar 13 observations and corresponding calibrations collected in HR-9 setup (514.3-535.4 nm). SNR is the average signal-to-noise ratio for star of $V$-magnitude $=18$.

\begin{tabular}{llcccccc}
\hline \hline$\#$ & Archives file name & $\begin{array}{c}\text { Date of } \\
\text { observations }\end{array}$ & $\begin{array}{c}\text { Time } \\
{[\mathrm{UT}]}\end{array}$ & $\begin{array}{c}\text { Instrument } \\
\text { configuration }\end{array}$ & $\begin{array}{c}\text { Exp. time } \\
{[\mathrm{s}]}\end{array}$ & $\begin{array}{c}\text { SNR } \\
\text { at } V=18\end{array}$ & Remark \\
\hline 1 & FLAMES_GIRAF_OBS244_0015 & $2002-09-01$ & $05: 29$ & Medusa2 & 3600 & 10 & good \\
2 & FLAMES_GIRAF_OBS244_0016 & $2002-09-01$ & $06: 45$ & Medusa1 & 3600 & 10 & $\begin{array}{c}\text { acceptable } \\
\text { poor }\end{array}$ \\
3 & FLAMES_GIRAF_OBS246_0003 & $2002-09-03$ & $03: 41$ & Medusa2 & 3600 & 7 & flat \\
\hline 4 & FLAMES_GIRAF_FLAT240_0008 & $2002-08-28$ & $19: 51$ & Medusa1 & 143 & ThAr \\
5 & FLAMES_GIRAF_WAVE240_0011 & $2002-08-28$ & $20: 10$ & Medusa1 & 484 & flat \\
6 & FLAMES_GIRAF_FLAT240_0020 & $2002-08-28$ & $23: 53$ & Medusa2 & 143 & ThAr \\
7 & FLAMES_GIRAF_WAVE238_0018 & $2002-08-26$ & $19: 33$ & Medusa2 & 482 & &
\end{tabular}

master localization and PSF model. It is worth mentioning that the final translation of the master wavelength solution, which is below $0.001 \mathrm{~nm}$ for all three science CCD frames, indicates an excellent instrument stability, at least over the period of one week spanning the above acquisition of data.

\subsection{Radial velocities}

Radial velocities $V_{\mathrm{r}}$ were then measured through the crosscorrelation of the above spectra with the standard GIRAFFE mask for the HR-9 setup. It is a binary CORAVEL-type mask for the stellar spectral type F0, with variable line-width ranging from 0.005 to $0.06 \mathrm{~nm}$ and a total number of 98 spectral lines. In this paper, the new FLAMES data are labeled with $F$ for FLAMES while the data from Côté et al. are labeled with $K$ for Keck. Table 2 summarizes our results for all observed stars. Columns (1) and (2) give the star identification number and its cluster membership probability, first from this FLAMES study and second from the Keck study, whenever available. All our stellar IDs come from Siegel et al. (2001). Column (3) through (6) give the equatorial coordinates $\alpha$ and $\delta$, the distance $R$ of the star to the cluster center $\left(\alpha_{\mathrm{J} 2000}=23^{\mathrm{h}} 06^{\mathrm{m}} 44.480\right.$, $\left.\delta_{\mathrm{J} 2000}=12^{\circ} 46^{\prime} 19^{\prime} .20\right)$ and the $V$ magnitude from Siegel et al. (2001). Column (7) through (9) give the radial velocities, whenever available, from each of our three FLAMES epochs. Column (10) gives for each star the number $n$ of valid measurements available from our three epochs, (11) indicates the crosscorrelation-peak quality $Q$ ( 1 for best and 9 for worst), where the first number represents the certainty of the peak identification and the second the quality of the fit, and (12) gives the standard deviation of the two or three measurements, whenever applicable. Column (13) gives the FLAMES mean radial velocity from our $n$ measurements and the estimated error $\left(\sigma_{V_{\mathrm{r}}}\right.$ or individual error) and Col. (13) is the Keck mean radial velocity from Côté et al. for comparison.

The raw measurements of 55 stars were cleaned by removing all measurements with unclear peak identification or very bad quality of the fit. From originally 55 objects, 46 remain with at least one valid radial velocity measurement. We get 4 objects with all three measurements, 30 objects with two measurements and 12 objects with one measurement ( 6 from first plate, 5 from second and 1 from third plate).

Ignoring the three $V_{\mathrm{r}}$ variable stars \#36 \#38 and \#156 (see discussion below), we have an immediate $V_{\mathrm{r}}$ quality check for the $27+4=31$ stars with at least two measurements: the mean of their individual standard deviations is $0.54 \mathrm{~km} \mathrm{~s}^{-1}$.

We also include in Table 2 the 12 stars with one measurement only but having acceptable cross-correlation-peak quality $Q$ (Col. 11). Note that measurements with $Q$ values higher than 2-3 should be considered with caution.

Figures 1 and 2 give the finding charts of all 46 stars measured with FLAMES. In Table 2, all stars are sorted by increasing values of their mean radial velocities listed in Col. (13). In this table, the intermediate horizontal line isolates the 11 stars (at the bottom of Table 2) with very similar radial velocities: they may be considered as cluster members when using the radial-velocity membership criterion only (see below).

It may be worth mentioning that all radial velocities listed in Cols. (7)-(9) are raw velocities, obtained directly from the cross-correlation process, corrected only for the solar system and earth velocities. No other attempt is made to correct for any effect due to various dependence on stellar spectral types (the same cross-correlation mask was used for all spectra) or any effect due to the limited spectral range. The undergoing analysis of other FLAMES data sets not related to Palomar 13 indicates that the random error due to the mask mismatch could be a few $0.1 \mathrm{~km} \mathrm{~s}^{-1}$ while the systematic shifts due to the limited wavelength coverage do not exceed a similar value (a few $0.1 \mathrm{~km} \mathrm{~s}^{-1}$ ) while using the HR-9 setup.

\section{Memberships}

\subsection{Membership from radial velocities}

A first glance at Col. (13) of Table 2 reveals immediately a clump of 11 stars whose radial velocities accumulate around a velocity close to the systemic radial velocity of Palomar 13, namely $24.1 \pm 0.5 \mathrm{~km} \mathrm{~s}^{-1}$, as measured by Côté et al. This clustering of radial velocities is significant: the $V_{\mathrm{r}}$ of all the other stars in Table 2 are away from the above systemic radial velocity by more than 10 times the standard deviation of the $V_{\mathrm{r}}$ of these 11 stars.

These 11 stars are potential cluster members, but before computing any new estimates of the systemic radial velocity and projected intrinsic velocity dispersion of Palomar 13, we first compare our measurements with the Keck radial velocities and check for possible non-members using proper motion and photometric data. 


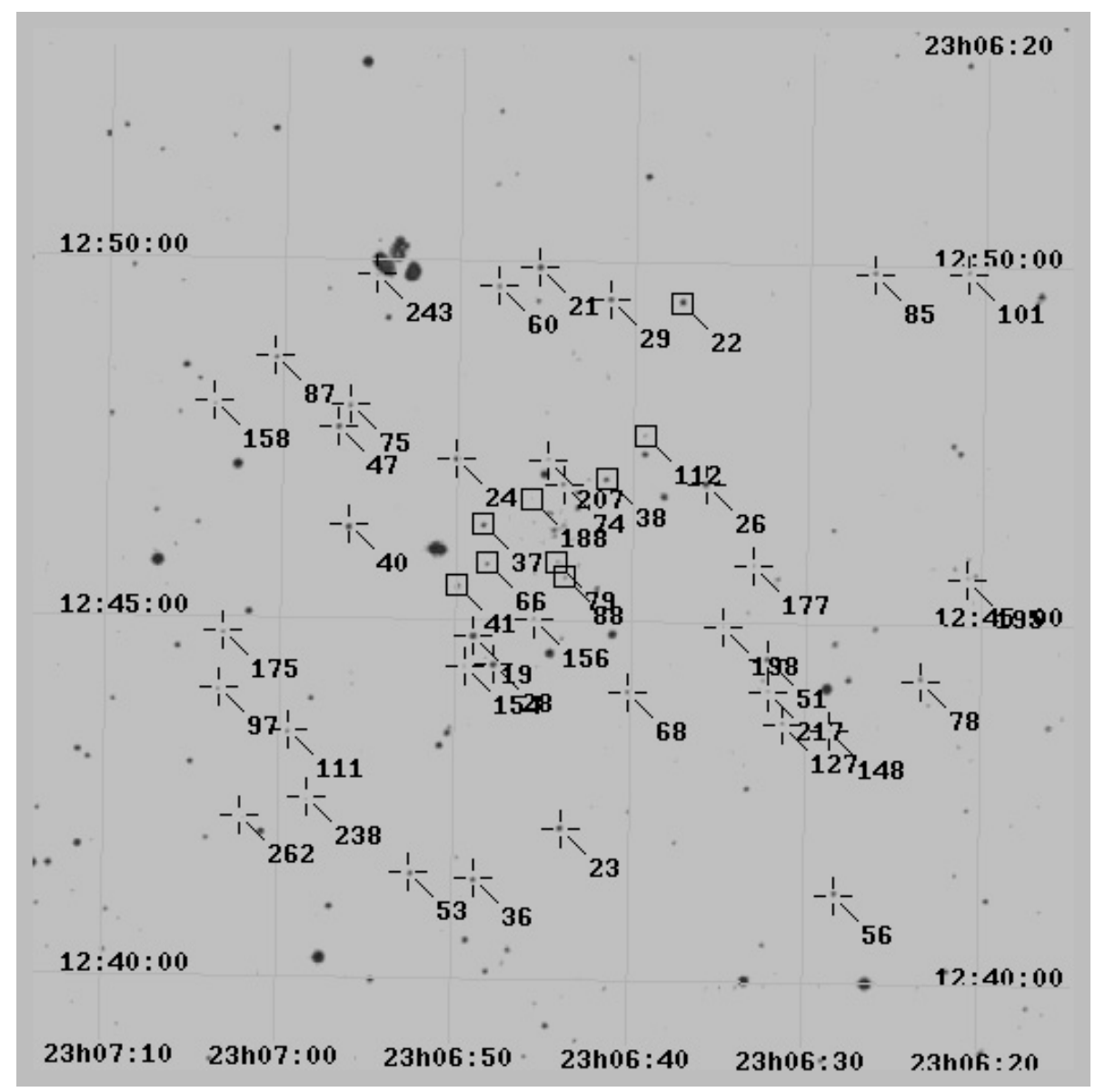

Fig. 1. Digital Sky Survey image with all 46 stars listed in Table 2. Stars are numbered according to Siegel et al. (2001). Member stars are enclosed by boxes and other program stars indicated with crosshairs. The entire FLAMES field (diameter $26^{\prime}$ ) is shown. See Fig. 2 for a zoom into the cluster center.

\subsection{Agreement with Keck radial velocities}

The last two columns in Table 2 provide, whenever available, i.e. for 13 stars, the radial velocities from both FLAMES $\left(V_{\mathrm{r}} \mathrm{FLAMES}\right)$ and Keck $\left(V_{\mathrm{r}}\right.$ Keck) studies. The global agreement is excellent. From the 13 stars, 9 lie within the limits $\mid V_{\mathrm{r}}$ FLAMES $-V_{\mathrm{r}} \mathrm{Keck} \mid<1.2 \mathrm{~km} \mathrm{~s}^{-1}$ with an average difference of $0.29 \mathrm{~km} \mathrm{~s}^{-1}$.

From the six stars with both FLAMES and Keck measurements, which are clearly non-member (above the intermediate horizontal line in Table 2), only one, with FLAMES ID \#156 (Keck ID \#23), has a radial velocity difference between two FLAMES epochs which is larger than the above $1.2 \mathrm{~km} \mathrm{~s}^{-1}$ limit. This star, clearly detected as variable through our internal consistency check, has a mean $V_{\mathrm{r}}$ FLAMES = $7.79 \pm 2.11 \mathrm{~km} \mathrm{~s}^{-1}$ (mean of two measurements) and a $V_{\mathrm{r}}$ Keck $=18.57 \pm 0.82 \mathrm{~km} \mathrm{~s}^{-1}$ (one measurement), with $\left|\Delta V_{\mathrm{r}}\right|=$ $12.27 \mathrm{~km} \mathrm{~s}^{-1}$. In both studies, this star is not considered as a cluster member, neither from proper motions nor from ColorMagnitude-Diagram (CMD) criteria. We consider this star as variable according to radial velocity (either pulsating or binary).
The situation is slightly different for the seven potential cluster members (below the intermediate horizontal line in Table 2) having both FLAMES and Keck measurements.

- The star with FLAMES ID \#66 (Keck ID \#41) has a $V_{\mathrm{r}} \mathrm{FLAMES}=26.69 \pm 0.5 \mathrm{~km} \mathrm{~s}^{-1}$ and a $V_{\mathrm{r}}$ Keck $=19.24 \pm$ $0.47 \mathrm{~km} \mathrm{~s}^{-1}$, with $\left|\Delta V_{\mathrm{r}}\right|=7.45 \mathrm{~km} \mathrm{~s}^{-1}$. In both studies, this star is considered as a cluster member. It is interesting to note that the above Keck value is the mean of three individual measurements, spread over more than a year, with $V_{\mathrm{r}}$ Keck $=18.91 \pm 0.93 \mathrm{~km} \mathrm{~s}^{-1}, 18.37 \pm 1.84 \mathrm{~km} \mathrm{~s}^{-1}$, and $19.43 \pm 0.54 \mathrm{~km} \mathrm{~s}^{-1}$, in chronological order. They do not show any significant variation.

- There are two more stars with marginal disagreements: first, stars with FLAMES ID \#37 (Keck ID \#72) has a $V_{\mathrm{r}}$ FLAMES $=26.32 \pm 0.2 \mathrm{~km} \mathrm{~s}^{-1}$ and a $V_{\mathrm{r}} \mathrm{Keck}=28.79 \pm$ $0.27 \mathrm{~km} \mathrm{~s}^{-1}$, with $\left|\Delta V_{\mathrm{r}}\right|=2.47 \mathrm{~km} \mathrm{~s}^{-1}$; second, star with FLAMES ID \#88 (Keck ID \#36) has a $V_{\mathrm{r}}$ FLAMES = $27.97 \pm 0.5 \mathrm{~km} \mathrm{~s}^{-1}$ and a $V_{\mathrm{r}} \mathrm{Keck}=25.29 \pm 0.89 \mathrm{~km} \mathrm{~s}^{-1}$, with $\left|\Delta V_{\mathrm{r}}\right|=2.68 \mathrm{~km} \mathrm{~s}^{-1}$.

Are these three stars pulsating or binaries? Two of them have only one FLAMES measurement, while the third star, with FLAMES ID \#37 has two FLAMES $V_{\mathrm{r}}$ measurements 


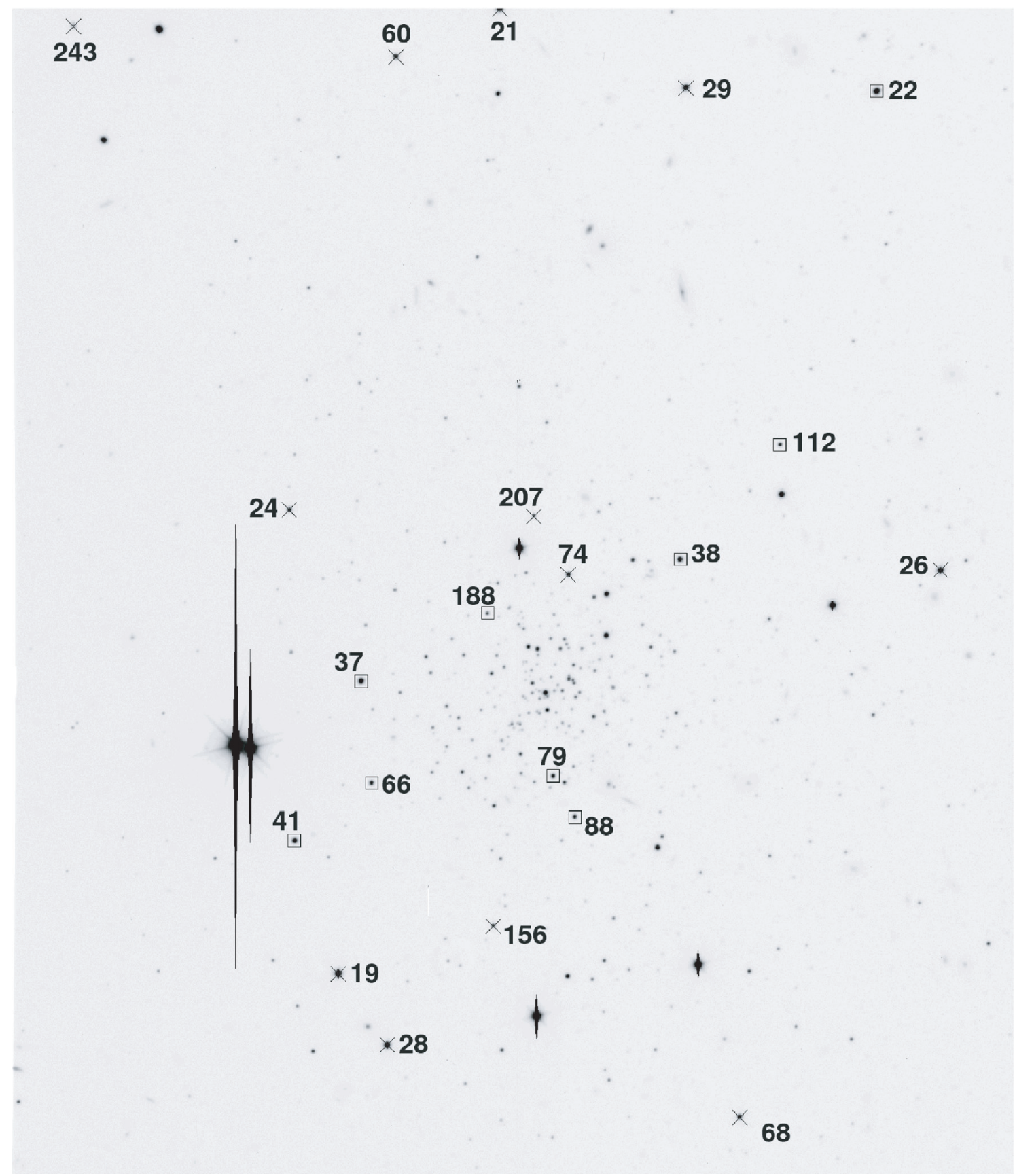

Fig. 2. Detailed finding chart of the central field: $V$-band image of Palomar 13 taken with the Low-Resolution Imaging Spectrometer on the Keck II telescope from Côté et al. (2002). Member stars are enclosed by boxes and other program stars indicated with crosses.

differing by $0.54 \mathrm{~km} \mathrm{~s}^{-1}$. We discuss below the influence of these stars on the velocity dispersion estimate of the cluster.

\subsection{One RR-Lyrae star in the cluster}

The star with FLAMES ID \#38 has two measurements, with $V_{\mathrm{r}}$ FLAMES $=35.87 \pm 0.70 \mathrm{~km} \mathrm{~s}^{-1}$ and $6.92 \pm 1.10 \mathrm{~km} \mathrm{~s}^{-1}$, with $\left|\Delta V_{\mathrm{r}}\right|=28.95 \mathrm{~km} \mathrm{~s}^{-1}$. This is a known RR-Lyrae variable (\#3 in Ciatti et al. 1965), however we ignore it in the remaining of this study in spite of the fact that it is considered as a cluster member.

\subsection{Membership from stellar proper motions and photometry}

Table 3 gives for the clump of 11 stars (at the bottom of Table 2), all of them potential cluster members through their $V_{\mathrm{r}}$, the following information (from Siegel et al. 2001). Columns (1)-(3) give the ID number and the equatorial coordinates $\alpha$ and $\delta$. Columns (4)-(6) provide the proper motion values $\mu(\alpha), \mu(\delta)$, and their related membership probability in \%. Column (7) gives the FLAMES radial velocity. Columns (8)-(10) display the $U, B, V$ photometry, while Col. (11) indicates the position of the star on the CMD: RRL for RR-Lyrae pulsating star, RGB for Red-Giant Branch, SGB 
Table 2. Barycentric radial velocities for 46 stars in the field centered on Palomar 13. The extra horizontal line isolates a clump of 11 stars with radial velocities very close to the systemic radial velocity of the star cluster, potentially members of the cluster.

\begin{tabular}{|c|c|c|c|c|c|c|c|c|c|c|c|c|c|}
\hline $\begin{array}{c}\text { ID } \\
F-K \\
(1)\end{array}$ & $\begin{array}{c}\mathrm{M} \\
F-K \\
(2)\end{array}$ & $\begin{array}{c}\alpha_{\mathrm{J} 2000} \\
{\left[^{\circ}\right]} \\
(3)\end{array}$ & $\begin{array}{c}\delta_{\mathrm{J} 2000} \\
{\left[{ }^{\circ}\right]} \\
(4)\end{array}$ & $\begin{array}{c}R \\
{\left[{ }^{\prime \prime}\right]} \\
(5)\end{array}$ & $\begin{array}{c}V \\
{[\mathrm{mag}]} \\
(6)\end{array}$ & $\begin{array}{c}V_{\mathrm{r}} 1 \\
{\left[\mathrm{~km} \mathrm{~s}^{-1}\right]} \\
(7)\end{array}$ & $\begin{array}{c}V_{\mathrm{r}} 2 \\
{\left[\mathrm{~km} \mathrm{~s}^{-1}\right]} \\
(8)\end{array}$ & $\begin{array}{c}V_{\mathrm{r}} 3 \\
{\left[\mathrm{~km} \mathrm{~s}^{-1}\right]} \\
(9)\end{array}$ & (10) & (11) & $\begin{array}{c}\sigma_{V_{\mathrm{r}}} \\
{\left[\mathrm{km} \mathrm{s}^{-1}\right]} \\
(12)\end{array}$ & $\begin{array}{c}V_{\mathrm{r}} \text { FLAMES } \\
{\left[\mathrm{km} \mathrm{s}^{-1}\right]} \\
(13)\end{array}$ & $\begin{array}{c}V_{\mathrm{r}} \text { Keck } \\
{\left[\mathrm{km} \mathrm{s}^{-1}\right]} \\
(14)\end{array}$ \\
\hline 29 & 0 & 346.67271 & 12.82458 & 190 & 17.33 & -248.39 & -248.12 & $\ldots$ & 2 & 11 & 0.19 & $-248.25 \pm 0.19$ & $\ldots$ \\
\hline 21 & 0 & 346.68958 & 12.83175 & 210 & 16.91 & -153.00 & -154.25 & $\ldots$ & 2 & 11 & 0.88 & $-153.62 \pm 0.88$ & ... \\
\hline 111 & 0 & 346.74825 & 12.72392 & 282 & 19.14 & $\ldots$ & -109.18 & $\ldots$ & 1 & 12 & $\ldots$ & $-109.18 \pm 1.27$ & $\ldots$ \\
\hline 101 & 0 & 346.58725 & 12.83158 & 410 & 19.13 & -66.55 & $\ldots$ & -67.13 & 2 & 13 & 0.41 & $-66.84 \pm 0.41$ & ... \\
\hline 195 & 0 & 346.58687 & 12.76064 & 356 & 19.85 & $\ldots$ & $\ldots$ & -61.50 & 1 & 23 & $\ldots$ & $-61.50 \pm 1.31$ & $\ldots$ \\
\hline 175 & 0 & 346.76379 & 12.74694 & 295 & 19.47 & -57.93 & $\ldots$ & $\ldots$ & 1 & 23 & $\ldots$ & $-57.93 \pm 2.01$ & $\ldots$ \\
\hline $26-103$ & $0-0$ & 346.64942 & 12.78197 & 133 & 17.05 & -57.38 & -57.19 & -58.54 & 3 & 11 & 0.73 & $-57.70 \pm 0.73$ & -56.72 \\
\hline 78 & 0 & 346.59775 & 12.73714 & 338 & 18.66 & -54.43 & $\ldots$ & -53.68 & 2 & 12 & 0.53 & $-54.05 \pm 0.53$ & $\ldots$ \\
\hline 40 & 0 & 346.73433 & 12.77156 & 176 & 17.27 & -45.06 & -45.47 & $\ldots$ & 2 & 11 & 0.28 & $-45.26 \pm 0.28$ & ... \\
\hline 56 & 0 & 346.61792 & 12.68717 & 384 & 18.21 & -42.36 & $\ldots$ & -42.88 & 2 & 11 & 0.36 & $-42.62 \pm 0.36$ & $\ldots$ \\
\hline $19-13$ & $0-0$ & 346.70421 & 12.74631 & 112 & 16.54 & -37.12 & -38.32 & $\ldots$ & 2 & 11 & 0.85 & $-37.72 \pm 0.85$ & -38.90 \\
\hline $68-1$ & $0-0$ & 346.66737 & 12.73358 & 149 & 18.57 & -36.66 & -37.61 & $\ldots$ & 2 & 12 & 0.67 & $-37.13 \pm 0.67$ & -37.48 \\
\hline 74-101 & $0-0$ & 346.68329 & 12.78169 & 34 & 18.64 & -18.23 & -17.71 & $\ldots$ & 2 & 11 & 0.36 & $-17.97 \pm 0.36$ & -17.39 \\
\hline 51 & 0 & 346.63421 & 12.74147 & 212 & 18.12 & -16.48 & $\ldots$ & -17.05 & 2 & 11 & 0.41 & $-16.77 \pm 0.41$ & $\ldots$ \\
\hline 177 & 0 & 346.63758 & 12.76325 & 174 & 19.61 & -11.92 & $\ldots$ & -10.80 & 2 & 12 & 0.80 & $-11.36 \pm 0.80$ & $\ldots$ \\
\hline 47 & 0 & 346.73692 & 12.79464 & 202 & 17.90 & $\ldots$ & -10.12 & $\ldots$ & 1 & 12 & $\ldots$ & $-10.12 \pm 1.34$ & $\ldots$ \\
\hline 243 & 0 & 346.72821 & 12.83008 & 255 & 20.14 & -9.01 & $\ldots$ & $\cdots$ & 1 & 23 & $\cdots$ & $-9.01 \pm 1.50$ & ... \\
\hline 97 & 0 & 346.76483 & 12.73361 & 316 & 19.14 & -5.21 & -4.63 & $\ldots$ & 2 & 23 & 0.41 & $-4.92 \pm 0.41$ & $\ldots$ \\
\hline 75 & 0 & 346.73433 & 12.79969 & 201 & 18.64 & -3.04 & -2.92 & $\ldots$ & 2 & 33 & 0.08 & $-2.98 \pm 0.08$ & $\ldots$ \\
\hline 158 & 0 & 346.76642 & 12.80003 & 308 & 19.53 & -1.00 & -0.81 & $\ldots$ & 2 & 23 & 0.13 & $-0.91 \pm 0.13$ & $\ldots$ \\
\hline 53 & 0 & 346.71908 & 12.69136 & 308 & 18.14 & 0.10 & $\ldots$ & -1.10 & 2 & 23 & 0.86 & $-0.50 \pm 0.86$ & ... \\
\hline 87 & 0 & 346.75204 & 12.81072 & 275 & 18.85 & $\ldots$ & -0.03 & $\ldots$ & 1 & 13 & $\cdots$ & $-0.03 \pm 0.97$ & $\ldots$ \\
\hline $28-6$ & $0-0$ & 346.69946 & 12.73983 & 123 & 17.02 & 0.69 & 1.32 & $\ldots$ & 2 & 11 & 0.44 & $1.01 \pm 0.44$ & 0.68 \\
\hline 207 & 0 & 346.68692 & 12.78711 & 53 & 19.91 & 1.49 & 2.70 & $\ldots$ & 2 & 24 & 0.86 & $2.10 \pm 0.86$ & ... \\
\hline 262 & 0 & 346.75937 & 12.70397 & 357 & 20.17 & 4.48 & 3.71 & 4.66 & 3 & 23 & 0.50 & $4.28 \pm 0.50$ & $\ldots$ \\
\hline 198 & 0 & 346.64487 & 12.74894 & 166 & 19.91 & $\ldots$ & 3.92 & 5.18 & 2 & 23 & 0.88 & $4.55 \pm 0.88$ & $\ldots$ \\
\hline 148 & 0 & 346.61904 & 12.72508 & 290 & 19.86 & $\ldots$ & 5.15 & 4.40 & 2 & 44 & 0.53 & $4.78 \pm 0.53$ & $\ldots$ \\
\hline 238 & 0 & 346.74342 & 12.70831 & 306 & 20.08 & 5.19 & 5.96 & $\ldots$ & 2 & 33 & 0.55 & $5.58 \pm 0.55$ & ... \\
\hline 217 & 0 & 346.63392 & 12.73411 & 227 & 19.97 & $\ldots$ & 6.05 & $\ldots$ & 1 & 13 & $\cdots$ & $6.05 \pm 1.71$ & $\ldots$ \\
\hline 154 & 0 & 346.70625 & 12.73928 & 137 & 19.71 & $\ldots$ & 7.07 & $\ldots$ & 1 & 23 & $\ldots$ & $7.07 \pm 1.25$ & ... \\
\hline 85 & 0 & 346.60979 & 12.83125 & 342 & 18.80 & 7.83 & $\ldots$ & 7.61 & 2 & 12 & 0.16 & $7.72 \pm 0.16$ & $\ldots$ \\
\hline $156-23$ & $0-0$ & 346.68996 & 12.75047 & 77 & 19.50 & 6.30 & 9.28 & $\ldots$ & 2 & 13 & 2.11 & $7.79 \pm 2.11$ & 18.57 \\
\hline 36 & 0 & 346.70350 & 12.68986 & 295 & 17.64 & 5.65 & 10.15 & $\ldots$ & 2 & 12 & 3.19 & $7.90 \pm 3.19$ & $\cdots$ \\
\hline 60 & 0 & 346.69912 & 12.82744 & 200 & 18.44 & 9.58 & 10.55 & $\ldots$ & 2 & 12 & 0.68 & $10.06 \pm 0.68$ & ... \\
\hline 23 & 0 & 346.68304 & 12.70192 & 246 & 16.97 & 11.71 & 12.03 & 11.19 & 3 & 11 & 0.42 & $11.64 \pm 0.42$ & $\ldots$ \\
\hline 38 & 1 & 346.67312 & 12.78292 & 58 & 17.81 & 35.87 & 6.92 & $\ldots$ & 2 & 12 & 20.47 & $21.39 \pm 20.47$ & $\ldots$ \\
\hline $112-910$ & $1-1$ & 346.66404 & 12.79308 & 106 & 19.28 & 24.40 & $\ldots$ & 24.33 & 2 & 24 & 0.05 & $24.36 \pm 0.05$ & 23.77 \\
\hline 22 & 1 & 346.65546 & 12.82411 & 212 & 16.90 & 24.80 & 24.84 & 23.60 & 3 & 12 & 0.71 & $24.41 \pm 0.71$ & $\ldots$ \\
\hline 24-118 & $1-0$ & 346.70879 & 12.78747 & 100 & 17.00 & 25.12 & 25.66 & $\ldots$ & 2 & 11 & 0.38 & $25.39 \pm 0.38$ & 24.92 \\
\hline $41-31$ & $1-1$ & 346.70812 & 12.75797 & 95 & 17.80 & 25.70 & 25.82 & $\cdots$ & 2 & 11 & 0.08 & $25.76 \pm 0.08$ & 25.09 \\
\hline 79 & 1 & 346.68458 & 12.76372 & 29 & 19.02 & 25.85 & $\ldots$ & 25.67 & 2 & 13 & 0.13 & $25.76 \pm 0.13$ & $\ldots$ \\
\hline 188-96 & $1-1$ & 346.69067 & 12.77817 & 28 & 19.83 & 25.96 & ... & $\ldots$ & 1 & 23 & $\ldots$ & $25.96 \pm 1.01$ & 25.38 \\
\hline $37-72$ & $1-1$ & 346.70212 & 12.77217 & 60 & 17.62 & 26.59 & 26.05 & $\ldots$ & 2 & 11 & 0.38 & $26.32 \pm 0.38$ & 28.79 \\
\hline 127 & 1 & 346.63037 & 12.72667 & 253 & 19.30 & 26.44 & $\ldots$ & $\ldots$ & 1 & 13 & $\ldots$ & $26.44 \pm 0.42$ & $\ldots$ \\
\hline $66-41$ & $1-1$ & 346.70117 & 12.76311 & 64 & 18.58 & 26.69 & $\cdots$ & $\cdots$ & 1 & 12 & $\cdots$ & $26.69 \pm 0.46$ & 19.24 \\
\hline $88-36$ & $1-1$ & 346.68258 & 12.76011 & 42 & 18.97 & 27.97 & $\ldots$ & $\ldots$ & 1 & 13 & $\ldots$ & $27.97 \pm 0.54$ & 25.29 \\
\hline
\end{tabular}

for Sub-Giant Branch, BS for Blue Straggler, and off-r for being off to the right of the cluster sequences.

The membership probabilities as determined by Siegel et al. (2001) are based on the proper motion relative to the average proper motion of the predetermined cluster members. The probability is computed using, for each individual candidate star, the ratio between the relative proper motion $\mu$ and the measurement error $\sigma_{\mu}$. The process is iterated till a clean sample of cluster members covering the area of study is obtained.

The proper motion membership probabilities in Table 3 give 8 stars out of 11 as clear members of the cluster, while the 2 stars with FLAMES ID \#24 and \#127 have zero 
Table 3. Membership probabilities from proper motions and positions in the CMD for the 11 stars with radial velocities implying membership of Palomar 13.

\begin{tabular}{|c|c|c|c|c|c|c|c|c|c|c|}
\hline (1) & $\begin{array}{c}\alpha_{\mathrm{J} 2000} \\
{\left[\begin{array}{c}\mathrm{h} \mathrm{m} \mathrm{s} \\
(2)\end{array}\right.} \\
(2)\end{array}$ & 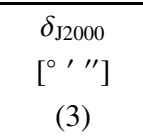 & $\begin{array}{c}\mu(\alpha) \\
{\left[\operatorname{mas~yr}^{-1}\right]} \\
(4)\end{array}$ & $\begin{array}{c}\mu(\delta) \\
{\left[\mathrm{mas} \mathrm{yr}^{-1}\right]} \\
(5)\end{array}$ & $\begin{array}{c}\mathrm{P}(\mu) \\
{[\%]} \\
(6)\end{array}$ & $\begin{array}{c}V_{\mathrm{r}} \text { FLAMES } \\
{\left[\mathrm{km} \mathrm{s}^{-1}\right]} \\
(7)\end{array}$ & $\begin{array}{c}U \\
{[\mathrm{mag}]} \\
(8)\end{array}$ & $\begin{array}{c}B \\
{[\mathrm{mag}]} \\
(9)\end{array}$ & $\begin{array}{c}V \\
{[\mathrm{mag}]} \\
(10)\end{array}$ & CMD \\
\hline 38 & 230641.55 & 124658.5 & $0.32 \pm 0.11$ & $0.05 \pm 0.19$ & 48 & 21.39 & $18.432 \pm 0.020$ & $18.335 \pm 0.010$ & $17.808 \pm 0.007$ & RRL \\
\hline 112 & 230639.37 & 124735.1 & $-0.31 \pm 0$ & $0.59 \pm 0$ & 89 & 24.36 & $20.134 \pm 0.042$ & $20.070 \pm 0.021$ & $19.276 \pm 0.014$ & SGB \\
\hline 22 & 230637.31 & 124926.8 & $-0.22 \pm 0.07$ & $-0.02 \pm 0.10$ & 96 & 24.82 & $18.148 \pm 0.023$ & $17.761 \pm 0.012$ & $16.897 \pm 0.007$ & RGB \\
\hline 24 & 230650.11 & 124714.9 & $0.24 \pm 0$ & 16 & 0 & 25.39 & $18.334 \pm 0.020$ & $17.983=$ & & off-r? \\
\hline 41 & 230649.95 & 124528.7 & $-0.05 \pm 0.12$ & $-0.25 \pm 0$ & 79 & 25.76 & $18.854 \pm 0.022$ & $18.658 \pm 0.011$ & $17.795 \pm 0$ & RGB \\
\hline 79 & 230644.30 & 124549.4 & $0.22 \pm 0.16$ & $-0.36 \pm 0.18$ & 65 & 25.76 & $19.418 \pm 0.027$ & $19.386 \pm 0.014$ & $19.021 \pm 0.012$ & BS? \\
\hline 188 & 230645.76 & 124641.4 & $-0.18 \pm 0.20$ & $0.09 \pm 0.23$ & 99 & 25.96 & $20.535 \pm 0.058$ & $20.529 \pm 0.024$ & $19.834 \pm 0.018$ & SGB \\
\hline 37 & 230648.51 & 124619.8 & $0.00 \pm 0.09$ & $0.32 \pm 0.09$ & 88 & 26.32 & $18.757 \pm 0.023$ & $18.537 \pm 0.011$ & $17.625 \pm 0.007$ & RGB \\
\hline 127 & 230631.29 & 124336.0 & $-4.4 \pm 0.77$ & $-6.2 \pm 0.54$ & 0 & 26.44 & $21.740 \pm 0.185$ & $20.579 \pm 0.034$ & $19.305 \pm 0.018$ & off-r \\
\hline 66 & 230648.28 & 124547.2 & $-0.41 \pm 0.13$ & $0.03 \pm 0.14$ & 88 & 26.69 & $19.427 \pm 0.028$ & $19.361 \pm 0.014$ & $18.583 \pm 0.010$ & SGB \\
\hline 88 & 230643.82 & 124536.4 & $-0.30 \pm 0.18$ & $-0.20 \pm 0.20$ & 93 & 27.97 & $19.842 \pm 0.033$ & $19.727 \pm 0.016$ & $18.970 \pm 0.011$ & SGB \\
\hline
\end{tabular}

probability of being members. The RR-Lyrae, FLAMES ID \#38, with a low membership probability of $48 \%$ is ignored anyway.

The CMD position in Table 3 gives 8 stars out of 11 as clear members of the cluster, corresponding to the cluster RGB, SGB and BS sequences. Again, the same two stars with FLAMES ID \#24 and \#127 are off the cluster sequences, to the right of the CMD, although less significantly for the former than the latter. This confirms the selection based on the proper motion.

The star with FLAMES ID \#79 (not measured in the Keck study) has a CMD position indicating that it may be a blue straggler; given its $V_{\mathrm{r}}$ FLAMES $=25.76 \pm 0.65 \mathrm{~km} \mathrm{~s}^{-1}$ and its proper motion membership probability of $65 \%$, we consider it as a cluster member.

\subsection{Eight members of Palomar 13?}

The above discussion illustrates the danger of considering only radial velocities in order to select cluster members. The two stars with FLAMES ID \#24 and \#127 (the latter not measured in the Keck study) with $V_{\mathrm{r}}$ FLAMES $=26.44 \pm 0.42 \mathrm{~km} \mathrm{~s}^{-1}$ and $25.39 \pm 0.21 \mathrm{~km} \mathrm{~s}^{-1}$ respectively, have $V_{\mathrm{r}}$ values very close to the cluster systemic radial velocity. However, both proper motion probabilities and CMD positions make us consider them as non-members of the cluster.

From the examples above, it becomes obvious that to declare a star as a member or not of Palomar 13 represents a difficult task, which needs the compulsory simultaneous use of at least the three sorting processes available so far, viz radial velocities, proper motions, and positions in the CMD.

We are left with a sample of eight stars probable members of Palomar 13.

\section{Velocity dispersion and mass-to-light ratio}

Given the limited size of our sample which does not permit any attempt to discuss any concentric variation of any kinematic quantity, we focus here on the best estimate of the unbiased mean radial velocity dispersion for the whole sample. The crucial element is the subtraction of the random error of our sample and the error estimate of our velocity dispersion. We compute the velocity dispersion and the mass-to-light ratio in four different ways: (i) for the eight stars with at least one FLAMES $V_{\mathrm{r}}$ value; (ii) for the five stars with two FLAMES $V_{\mathrm{r}}$ values (measured twice), and then for the six stars which are measured in both Keck and FLAMES studies; (iii) for the six FLAMES $V_{\mathrm{r}}$ values and (iv) for the six Keck $V_{\mathrm{r}}$ values.

The average empirical variance of radial velocity of 31 stars with at least two measurements is $v^{2} r_{0}=0.29 \mathrm{~km}^{2} \mathrm{~s}^{-2}$. This is the most realistic estimate of the measurement error applicable to all measurements. The stars with two measurements are taken with var $=0.5$ var $_{0}$ while stars with only one measurement with var $=v a_{0}$. We compute the unbiased systemic cluster velocity $\left\langle V_{\mathrm{r}}\right\rangle$, the intrinsic projected cluster velocity dispersion $\sigma_{\mathrm{p}}$ and associated errors according to the method described in Pryor \& Meylan (1993), which were also used in the Keck study (Côté et al.).

\subsection{Sample of eight stars with at least one FLAMES $V_{r}$ value}

The systemic velocity of Palomar 13 from our eight member stars is $\left\langle V_{\mathrm{r} 8}\right\rangle=25.91 \pm 0.36 \mathrm{~km} \mathrm{~s}^{-1}$ with a projected velocity dispersion $\sigma_{\mathrm{p} 8}=0.92 \pm 0.29 \mathrm{~km} \mathrm{~s}^{-1}$. This $\sigma_{\mathrm{p} 8}$ value should be considered as an upper limit to the real value since, for a given sample, any source of unknown random error will tend to increase the measured velocity dispersion. The above value is significantly smaller than $\sigma_{\mathrm{p}}=2.2 \pm 0.4 \mathrm{~km} \mathrm{~s}^{-1}$ found by Côté et al.

Note that even a very crude unweighted computation of the systemic velocity and projected velocity dispersion will not produce significantly different results: namely, for the same eight stars, $\left\langle V_{\mathrm{r}}\right\rangle=25.95 \mathrm{~km} \mathrm{~s}^{-1}$ and $\sigma_{\mathrm{p}}=1.04 \mathrm{~km} \mathrm{~s}^{-1}$. In general terms, we can say that whatever the sophistication of the 
weighting process used in the above computation, the resulting velocity dispersion will always be close to $1 \mathrm{~km} \mathrm{~s}^{-1}$. This is a robust result.

What is the mass-to-light ratio corresponding to this new velocity dispersion $\sigma_{\mathrm{p}}$ value? If only the $\sigma_{\mathrm{p}}$ value changes, everything else being equal (King-Michie model, core radius, and central surface brightness, see Table 3 from Côté et al.), the value of the $\mathcal{M} / L_{V}$ ratio varies as the squared ratio of the old to new velocity dispersions. Consequently, the new $\mathcal{M} / L_{V}$ is equal to the old $\mathcal{M} / L_{V}$ divided by $(2.2 / 0.92)^{2}=5.8$, namely, $\mathcal{M} / L_{V}=40 / 5.8 \sim 7$, a value significantly closer to the usual value $\mathcal{M} / L_{V} \sim 3$ for globular clusters.

Since we have a rather small sample, we explore briefly the sensitivity of the above results to various subsamples.

\subsection{Sample of five stars with two FLAMES $V_{r}$ measurements}

Similar arithmetics applied to the five Palomar 13 member stars with at least two FLAMES measurements gives the values $\left\langle V_{\mathrm{r} 5}\right\rangle=25.40 \pm 0.32 \mathrm{~km} \mathrm{~s}^{-1}$ and $\sigma_{\mathrm{p} 5}=0.60 \pm 0.27 \mathrm{~km} \mathrm{~s}^{-1}$. This again may be considered as an upper limit to the real value. From $(2.2 / 0.60)^{2}=13.4$, the new $\mathcal{M} / L_{V}=40 / 13.4 \sim 3.0$, a value identical to the usual value for globular clusters.

\subsection{Sample of six stars with FLAMES and Keck $V_{r}$ measurements}

Six of the above eight Palomar 13 stars have $V_{\mathrm{r}}$ measurements from both FLAMES and Keck studies.

The six $V_{\mathrm{r}}$ measurements from this FLAMES study bring the values $\left\langle V_{\mathrm{r} 6 \mathrm{~F}}\right\rangle=26.14 \pm 0.44 \mathrm{~km} \mathrm{~s}^{-1}$ and $\sigma_{\mathrm{p} 6 \mathrm{~F}}=$ $0.99 \pm 0.34 \mathrm{~km} \mathrm{~s}^{-1}$. The corresponding $\mathcal{M} / L_{V}$ would scale by $(2.2 / 0.99)^{2}=4.9$, namely, $\mathcal{M} / L_{V}=40 / 4.9 \sim 8$.

The six $V_{\mathrm{r}}$ measurements from the Keck study bring the values $\left\langle V_{\mathrm{r} 6 \mathrm{~K}}\right\rangle=24.60 \pm 1.16 \mathrm{~km} \mathrm{~s}^{-1}$ and $\sigma_{\mathrm{p} 6 \mathrm{~K}}=2.79 \pm 0.83 \mathrm{~km} \mathrm{~s}^{-1}$. The corresponding $\mathcal{M} / L_{V}$ would scale by $(2.2 / 2.79)^{2}=0.62$, namely, $\mathcal{M} / L_{V}=40 / 0.62 \sim 60$.

For the same stars, FLAMES $V_{\mathrm{r}}$ values produce a smaller velocity dispersion than the Keck $V_{\mathrm{r}}$ values, indication of a possible better stability and better zero-point calibration for the simultaneous FLAMES measurements than for the independent Keck ones.

\subsection{Overlap of Keck and FLAMES sample fields}

Because of the positioning of FLAMES fibres, which prevents the measurements of stars with small separations, the star sample acquired during this commissioning observing run is not optimal for the study of Palomar 13. We do not have numerous member stars within the tidal radius of the cluster as determined by Côté et al. Following our three criteria (radial velocities, proper motions, and CMD photometry), there seems to be members of Palomar 13 well outside the nominal tidal radius, as already noticed by Côté et al.

Could our small velocity dispersion value originate from the fact that we have stars on average more distant from the cluster center than those in the Keck study? This is not the case. We do not observe any central increase in velocity dispersion in Keck data since the stars contributing strongly to the velocity dispersion lie all outside the radius $60^{\prime \prime}$.

\section{Final remarks}

Although small, the present sample of radial velocities measured with FLAMES provides velocity dispersion and massto-light ratio estimates which differ significantly from those obtained from Keck radial velocities.

In both, FLAMES and Keck samples, the detection of binary stars is difficult. Côté et al. have good time coverage (up to four epoch) but few stars measured more than once, while FLAMES has two measurements for most stars but no time coverage. Two stars, \#66-41 and \#37-72 are responsible for significant differences between FLAMES and Keck results. Though these objects have not shown significant variability in, respectively, three and four Keck measurements and fall in the $V_{\mathrm{r}}$ range of the cluster in FLAMES data, we must accept them as binary candidates. Based on the proportion of blue stragglers in CMD of Palomar 13, approximately $25 \%$ of evolved stars are binary systems, a figure well compatible with two binaries found and simulations carried out by Côté et al.

The difference between FLAMES and Keck results casts doubt on the previous Keck velocity dispersion determination. The most simple and likely explanation of the very high Keck $\mathcal{M} / L_{V}$ ratio is the Keck sample contamination by binaries since the determination of velocity dispersion even from the larger (still small) sample from Keck is prone to be biased by the presence of few undected binaries.

A complete and more detailed analysis of these FLAMES radial velocities along with the old and some new radial velocities from Keck is under preparation, with extensive simulations of such observations. A careful attention will be given to the surface density profile of Palomar 13 which anyway appears to be anomalous among Galactic globular clusters. Is there anything genuinely peculiar with Palomar 13? A significant effort (new observations and new analysis) is needed to answer the following question: is Palomar 13 the host of a large fraction of binaries which may inflate its velocity dispersion? With a good observing strategy, FLAMES is currently the best instrument to answer this question.

Acknowledgements. We thank Pat Côté and S. G. Djorgovski for the image used in Fig. 2 as finding chart. We are very grateful to G. Simond for his work in the development of the GIRAFFE Data Reduction Software, G. Burki and M. Grenon for usefull discussions and the FLAMES commissioning team for the data. Part of this work was supported by Swiss Fonds National de la Recherche Scientifique.

\section{References}

Binney, J. 2003, [arXiv: astro-ph/0310219]

Blecha, A., Cayatte, V., North, P., Royer, F., \& Simond, G. 2000, Datareduction software for GIRAFFE, the VLT medium-resolution multi-object fiber-fed spectrograph, in Optical and IR Telescope Instrumentation and Detectors, ed. I. Masanori, \& A. F. Moorwood, SPIE, 4008,467 
Ciatti, F., Rosino, L., \& Sussi, M. 1965, Kl. Veroff. Remeis-Sternwarte Bamberg, 4, 40, 228

Côté, P., Djorgovski, S. G., Meylan, G., Castro, S., \& McCarthy, J. K. 2002, ApJ, 574, 783

Klypin, A. A., Kravtsov, A. V., Valenzuela, O., \& Prada, F. 1999, ApJ, 522,82

Meylan, G., \& Heggie, D. C. 1997, A\&ARv, 8, 1

Moore, B., Ghinga, S., Governato, F., et al. 1999, ApJ, 524, 19

Moore, B., Calcáneo-Roldán, C., Stadel, J., et al. 2001, Phys. Rev. D, 64, 063508

Pasquini, L., Avila, G., Blecha, A., et al. 2002, Installation and commissioning of FLAMES, the VLT Multifibre Facility, The Messenger, 110, 1
Pryor, C., \& Meylan, G. 1993, in The Structure and Dynamics of Globular Clusters, ed. S. G. Djorgovski, \& G. Meylan, ASP Conf. Ser., 50, 357

Royer, F., Blecha, A., North, P., et al. 2002, Toward accurate radial velocities with the fiber-fed GIRAFFE multi-object VLT spectrograph, in Astronomical Data Analysis II, ed. J.-L. Starck, \& F. D. Murtagh, SPIE, 4847, 184

Siegel, M. H., Majewski, S. R., Cudworth, K. M., \& Takamiya, M. 2001, AJ, 121, 935 